\title{
The detection of Chinese strokes and characters in visual noise
}

\author{
BO-LIN YU \\ Institute of Psychology, Chinese Academy of Sciences, Beijing, People's Republic of China
}

and

\author{
JOHN BROGAN, SUSAN ROBERTSON, and WILLIAM R. UTTAL \\ University of Michigan, Ann Arbor, Michigan
}

\begin{abstract}
This report discusses the effects of experience, method, and stimulus form on the detectability of dotted approximations to Chinese strokes and characters when they are masked by random. dot visual noise. The results of two experiments are interpreted to show that experience effects are nonexistent or small. Furthermore, even when small effects are present, they are in the direction opposite to that predicted. Similarly, method plays only a minor role in determining performance. However, strong stimulus-form effects exist that are well predicted by a previously developed autocorrelational-type computational model of the dotted form detection process.
\end{abstract}

Visual masking of dotted visual forms by random-dot visual noise is a useful means of assaying the properties of an intermediate level of visual processing (Uttal, 1975, $1983,1984)$ that is mediated by neither higher cognitive functions nor peripheral receptor mechanisms. An implicit assumption underlying this methodology is that the mechanisms under investigation are built into the visual system in a fixed fashion that is not susceptible to learning or experience with particular classes of dotted visual stimuli.

The purpose of the present study was threefold. First, it was to test this assumption of cognitive and experiential independence by examining dotted form detection in a situation in which the stimulus forms are heavily loaded with a culturally dependent symbolism. Specifically, we report the results of two experiments that measured the detectability of Chinese pictographic characters by two groups of observers-one a group of University of Michigan undergraduates who had had no experience with the Chinese language and the other a group of Chinese scholars at the University of Michigan who had had lifetime experience with these symbols.

A second and closely related purpose of this experiment was to test the applicability of a previously proposed (Uttal, 1975) model of visual form detection based on an autocorrelation-like transformation with a new set of stimulus forms-13 Chinese pictographs (4 single strokes and 9 characters) - for which both psychophysical detectability scores and computationally predicted figures of merit of detectability are obtained.

J. Brogan, S. Robertson, and W. R. Uttal were associated with the Perception Laboratory of the Institute for Social Science at the University of Michigan while this work was being conducted. Reprint requests should be sent to W. R. Uttal, who is now at the Naval Ocean Systems Center, Hawaii Laboratory, Box 997, Kailua, Hawaii 96734.
A third variable of concern to us dealt with a purely methodological matter. Two modes of stimulus presentation were used in this experiment-a dynamic mode in which dotted noise was presented serially over time and a static mode in which both the dotted stimulus form and the dotted noise were simultaneously and stably presented for the entire stimulus epoch. A comparison of the two data sets resulting from the two methods would provide an alternative measure of the period of persistence of the visual system.

\section{METHOD}

\section{Subjects}

The subjects in this experiment consisted of six members of the University of Michigan academic community. Three were undergraduate students in their early 20 s with no previous exposure to Chinese pictographic calligraphy, and three were students who were in their mid-30s and had been reading and writing the Chinese pictographs throughout their lives. All six subjects were paid an hourly stipend and participated throughout the course of the experiment. The two groups of three subjects were run on identical experimental protocols.

\section{Procedure}

The procedure used in this study was the same as that described in several publications in the past few years. The most complete description of the procedure is to be found in Uttal (1975). In brief, dotted stimulus forms (the approximations to the 13 Chinese strokes and characters shown in Figure 1) are presented on a CRT display observed simultaneously by both eyes (i.e., binocularly). The task is challenging because these dotted strokes and characters are degraded by being hidden within varying amounts of dotted visual noise. In general, it has previously been established that increasing the density of the noise decreases the detectability of the stimulus forms. Dotted visual noise is defined, for the purposes of this study, as an array of single punctuate lights distributed in random positions over the display surface.

The experiment was carried out in two modes. In the static mode, both the dots of the stimulus form and the randomly positioned noise dots are displayed rapidly enough to produce the appearance of a 

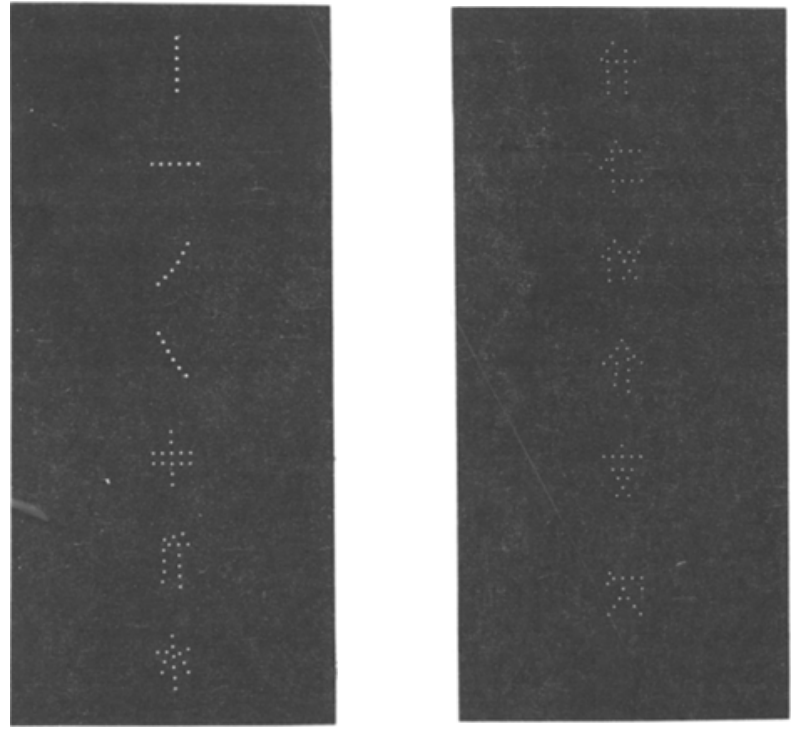

Figure 1. The 13 stimuli used in both parts of this experiment.

simultaneous display of all stimulus form and visual-noise dots. This produces a constant snapshot-like appearance of the masking and stimulus-form dots throughout the 1-sec display presentation period. In the dynamic mode, however, noise dots are presented sequentially, but at fixed interdot intervals that are large enough to allow perceptual nonsimultaneity. The persistence of the visual system, however, makes it appear that more than a single dot is present at any one instant. However, since previously plotted noise dots are fading and new dots are being added to the display constantly, the dynamic display mode has a twinkling, moving appearance during the entire epoch of the stimulus presentation. In this mode, the dots making up the stimulus form (the Chinese stroke or character) are presented as rapidly as possible (they last for less than $1 \mathrm{msec}$ ) and only once, halfway $(500 \mathrm{msec})$ through the 1 -sec-long presentation period.

The subject's task is to specify in which of two sequential presentations the stimulus form is presented. This task requires that the subject extract a particular constellation of organized dots (the stimulus form) from among a more extensive and (typically) denser array of randomly positioned visual noise dots. The organization of the dots in the Chinese stroke or character is, therefore, the sole variable distinguishing the constellation of dots making up the form from those making up the noise. A sample stimulus (comparable to a single static presentation) is shown in Figure 2.

Each trial consists of a fixed sequence of events, as diagramed in Figure 3. First, a fixation point is presented on the cathode ray tube display for $1 \mathrm{sec}$. This fixation point allows the subject to fixate the test region and to properly accommodate for the $33-\mathrm{cm}$ viewing distance. The first 1-sec stimulus presentation is then displayed. It may or may not (with a probability of $50 \%$ ) contain the stimulus form to be detected as well as the array of random masking dots. Following the first presentation, the fixation point is presented again for $1 \mathrm{sec}$ and then the second 1-sec-long stimulus presentation occurs. If the first presentation did not contain the stimulus form, then the second presentation does. In the presentation not containing the stimulus form, an additional number of random dots (the dummy dots) are added to the array of noise dots. (The noise array used in the two presentations of each trial is the same, but new maskingdot arrays are calculated for each trial.) The dummy dots are equal in number to the number of dots in the stimulus form but are randomly positioned within the area in which the form occurs. Thus, the two presentations are exactly equal in the number of dots, but differ in the geometrical organization of a subset of them-one of the two trials contains an ordered subarray-a Chinese character or stroke.

Following the second presentation, the display screen remains blank until the subject indicates his or her decision that the stroke or character occurred in either the first or second presentation by depressing one of two hand-held push buttons. A plus or a minus signal is then presented on the screen to tell the subject whether he or she was correct or incorrect. This feedback makes the entire sequence of trials a continuous training experience that builds upon

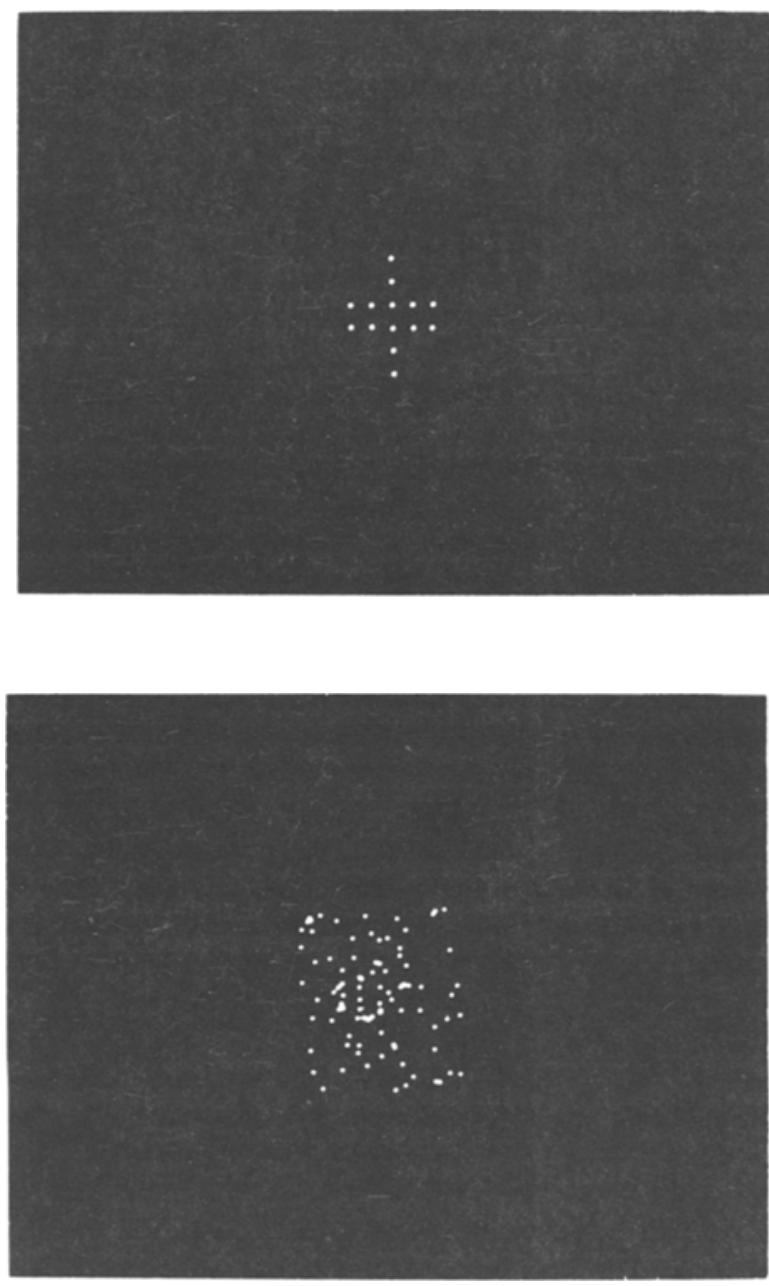

Figure 2. Stimulus number 5 shown without masking-noise and with masking-noise dots to illustrate the degradation occurring when it is so masked.

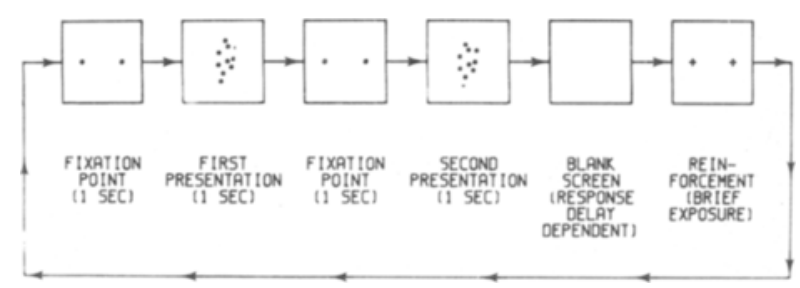

Figure 3. A flowchart showing the sequence of events in a single experimental trial. 
a block of initial training sessions. All subjects, prior to the actual data-collection sessions, participated in five 1-h sessions in which the stimulus forms and dummy dots were presented in their respective presentations, but with ineffectively small numbers of masking dots. All knew that the stimulus forms were approximations to Chinese characters and could easily recognize them when only a few masking dots were present.

\section{Instrumentation}

The entire sequence of events in each trial, as well as the entire experimental protocol, is presented automatically under computer control. Subjects arrive at the experimental room, sign on by depressing a few keys at the computer terminal, and are presented with a sequence of trials that is paced by their own response rates. Displays are presented on a cathode ray tube oscilloscope whose display surface is coated with a high-speed (P-24) phosphor with a decay time (to $1 \%$ of initial luminance) of only a few microseconds. The region in which the stimulus forms-the Chinese characters or strokes-occur is approximately $4^{\circ} \times 4^{\circ}$ in extent, depending on the particular stroke or character. The characters are embedded in noise, which subtends a somewhat larger area of $5.4^{\circ} \times 5.4^{\circ}$. The digitally encoded dotted Chinese characters and strokes are prepared prior to the experiment, using a two-dimension digitizer, and then stored in a disk storage file from which their coded representations are recalled to be linked with the operating portions of the computer control program. Two versions of the control program, one using static noise and one using dynamic noise, were prepared for the two experiments that are reported here.

At the end of an hour-long session, the subject signals the computer that the session is complete by typing in a special code character. The microcomputer program then branches to a data analysis program which automatically provides a preliminary tabulation of the results obtained in that session. A second data analysis program can be called by the experimenter to summarize the results of the sessions for all subjects for identical conditions and to provide more detailed analysis of the cumulative results.

The subject is isolated in a darkened, but not soundproofed, cubicle and has his or her head position restrained by a chinrest and forehead-controlled switch that inactivates the response switches unless the head is properly positioned.

\section{Experimental Protocol}

Two separate experiments were carried out in this study. One used the dynamic masking-dot mode, and the other used the static masking-dot mode, but both used the same set of 13 Chinese strokes and characters. The first experiment, which used dynamic noise, consisted of nine daily sessions in which the noise-dot density was progressively increased by selecting interdot intervals that varied from as long as $25 \mathrm{msec}$ to as short as $2 \mathrm{msec}$. Specifically, interdot intervals of $25,16,12,10,8,6,4,3$, and $2 \mathrm{msec}$ were used.

In the second experiment, in which static masking dots were used, only six masking-dot densities were used. In this case, the density of the masking dots is defined by the number of randomly dispersed dots in the $5.4^{\circ} \times 5.4^{\circ}$ viewing region. This density was increased in six sequential sessions using $40,62,83,100,125$, and 166 dots, respectively.

Approximately $\mathbf{5 0 0}$ trials are run in each $\mathbf{5 0}$-min session. Thus, depending on the nature of the analysis and the way in which data are pooled, as many as 1,500 trials, but no fewer than 230 trials, contribute to the scores indicated by each plotted data point. Because of these large numbers and the pooling of data into percentcorrect scores, formal tests of significance would not be meaningful.

\section{RESULTS}

The results of the two experiments-one using dynamic noise and one using static noise-will now be analyzed in several ways in this section to evaluate the influence of the three major variables of interest-Chinese-reading versus non-Chinese-reading subject experience, static and dynamic noise test conditions, and the specific form of the Chinese characters.

First let us consider the influence of intersubject differences. Figure 4 presents the data obtained from the two groups of subjects (Chinese reading and non-Chinese reading) for the dynamic-noise experimental test condition. All data from all of the 13 strokes and characters were pooled in this figure and the data plotted as a function of the interval between the dynamic-noise dots. In this case, there is a remarkable overlap of the two curvesboth Chinese-reading and non-Chinese-reading subjects performed identically.

Figure 5, on the other hand, indicates a somewhat different picture for the static-noise experiment. In this case, data for the two groups of subjects do not overlap as well. Rather, there is an irregular pattern of differences between the two groups. The Chinese-reading subjects are superior

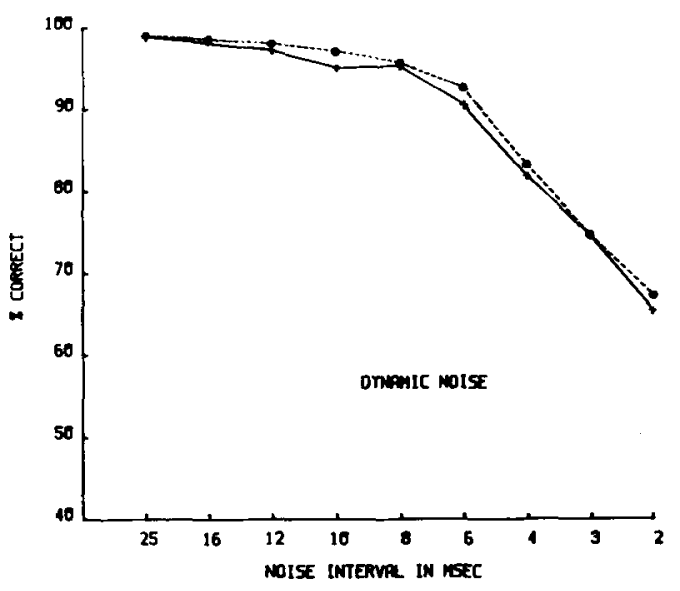

Figure 4. The results of the dynamic noise experiment. The two curves represent the results for the Chinese-reading $(+)$ and nonChinese-reading (o) subject groups, respectively.

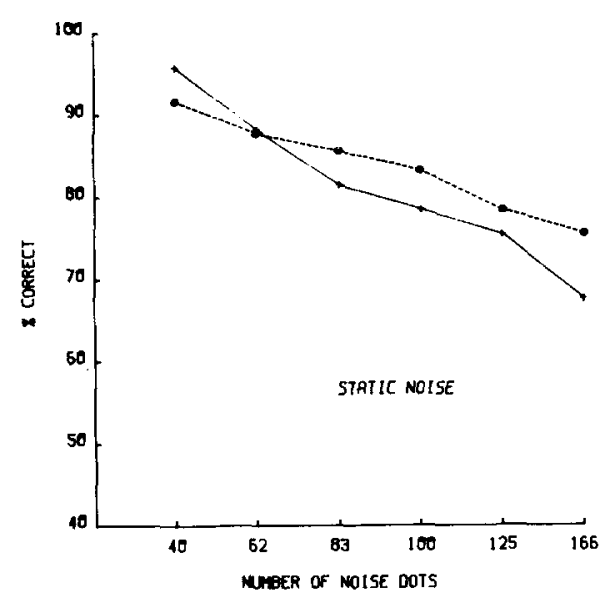

Figure 5.The results of static-noise experiment. The two curves represent the results for the Chinese-reading $(+)$ and non-Chinesereading (o) subject groups, respectively. 


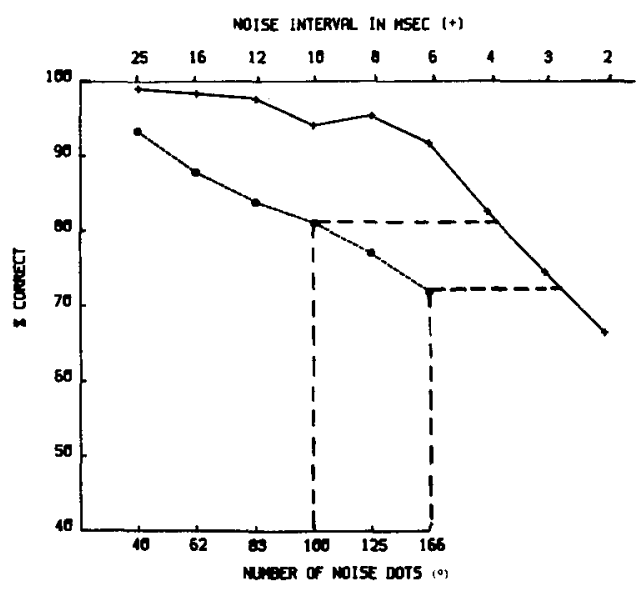

Figure 6. A comparison of the static and dynamic experiments. Data has been pooled for all six subjects. The two curves represent the results for the two different methods. The figure is used as a nomograph to calculate the effective visual persistence, as exemplified by the two L-shaped pairs of dotted lines and as explained in the text.

at the lowest noise density. The difference, however, disappears at 62 noise dots, only to reappear in favor of the non-Chinese-reading subjects for all higher noise-dot densities. Although these differences are not large (at most $8 \%$ and generally less than $5 \%$ ), they do present a picture different from that seen in Figure 4. At present, we have no definitive answer as to why the two groups differ, but suggest that it may be due to the age differential between them.

Second, let us consider the effect of the two modes of experimentation-static and dynamic. To make this comparison, all data-from all six subjects, for all noise levels, and for all Chinese characters-were pooled and plotted separately for each of the two experiments. The resulting two curves were plotted in Figure 6 as a function of the noise interval (in the dynamic case) and the number of noise dots (in the static case). The two curves in this figure can now be used as a nomograph to estimate the period of effective visual persistence in this type of experiment. To do so, we must proceed on the assumption that equal numbers of perceptually present noise dots produce equal masking effects in the two experiments. (This is a plausible, but admittedly unproven, hypothesis.) Comparing equal performance levels for the two curves, we can determine the dynamic noise interval that is equivalent to the static situation. Assuming the equivalence assumption is correct, by then multiplying the interval between the dynamic-noise dots by the number of static dots for equivalent performance levels, we can obtain an estimate of the effective period of visual presentation. A priori, any value of the static-noise density can be used as a criterion for this calculation equally as well as any other, and, as we shall see, the estimates obtained in each case differ from each other. Drawing horizontal lines between equivalent performance scores gives dynamic interval values of approximately $6.3 \mathrm{msec}$ for the 40 staticnoise-dot condition, $5.5 \mathrm{msec}$ for 62 noise dots, $4 \mathrm{msec}$ for 83 noise dots, $3.8 \mathrm{msec}$ for 100 noise dots, $3.2 \mathrm{msec}$ for 125 noise dots, and $2.7 \mathrm{msec}$ for 166 noise dots. Crossmultiplying these intervals and dot numerosities gives the following series of estimates of the effective persistence: $252,341,332,380,400$, and 448 at increasing static

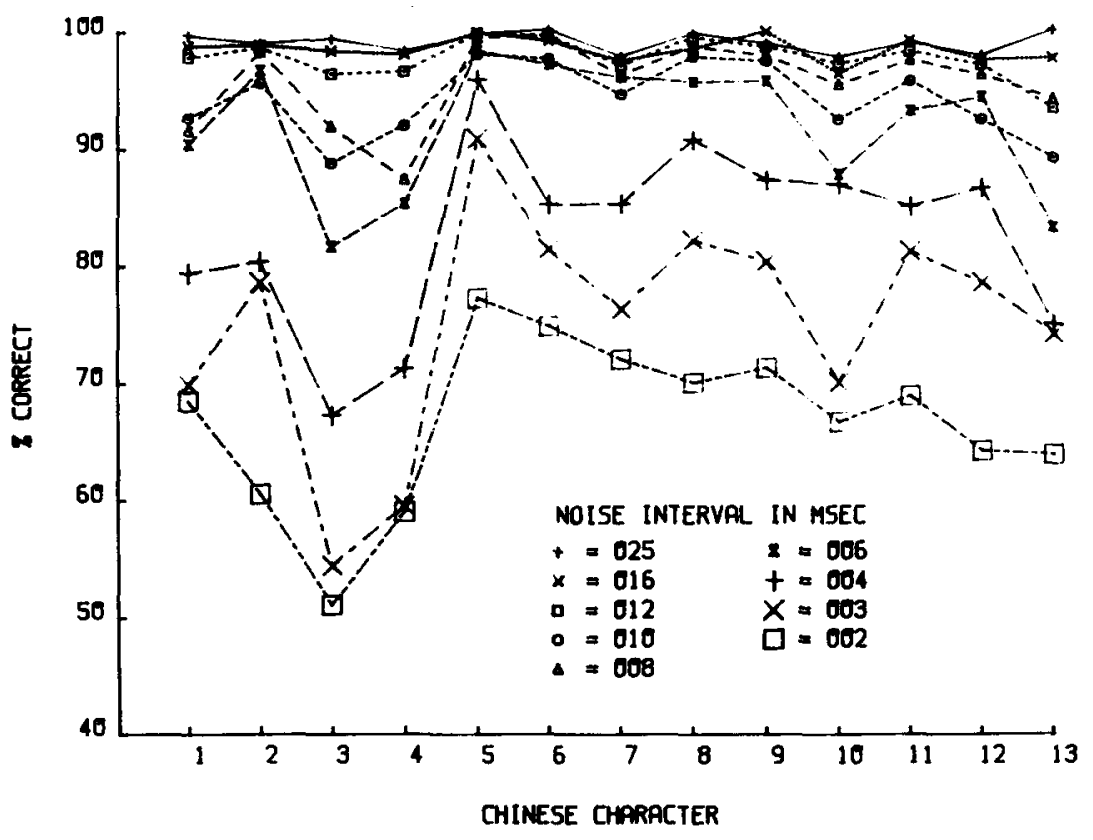

Figure 7. The results of the dynamic-noise experiment plotted as a function of the 13 characters or strokes. The set of curves is parametric as a function of the density of the noise dots as specified by the inter-noise-dot interval. 
noise-dot densities. Even given the uncertainties due to inter- and intrasubject variabilities, the general increasing order of these values is obvious.

The major concern in this study, however, was the effect of the form of the stimulus-the dotted approximations to the 13 Chinese characters or strokes shown in Figure 1-on detectability. Figures 7 and 8 display the results of the two experiments using dynamic and static noise, respectively. In each of these figures, data for all six subjects are pooled. The data were plotted parametrically as a function of either the dynamic-noise interval or the static-noise dot density.

In Figures 9 and 10, the two sets of data were pooled across the masking-dot interval or dot density for the dynamic and static experiments, respectively, and replotted to show the overall trend obtained in each experiment as a function of stimulus form. The most obvious conclusion to be drawn from these data is that the nature of the form effect is closely replicated in these figures. Although the absolute levels of performance in the dynamic-noise experiment are somewhat higher than the absolute levels of performance in the static-noise experiment (probably due to the inclusion of the several lower levels of noise density in the former case that produced no appreciable effect in this analysis), the relative detectability of virtually all of the characters and strokes is the same in both experiments. The few small reversals that exist are small enough to safely be considered to be examples of normal experimental variability.

Several points concerning these two graphs are of particular and immediate noteworthiness. First, there is only one character that stands out as being much more detectable than any of the others-the one numbered 5. This is so in spite of the fact that character 5 has the same number of dots as the other eight characters. Second, there are only two stimuli-the two curved strokes numbered 3 and 4-that are substantially lower in detectability than

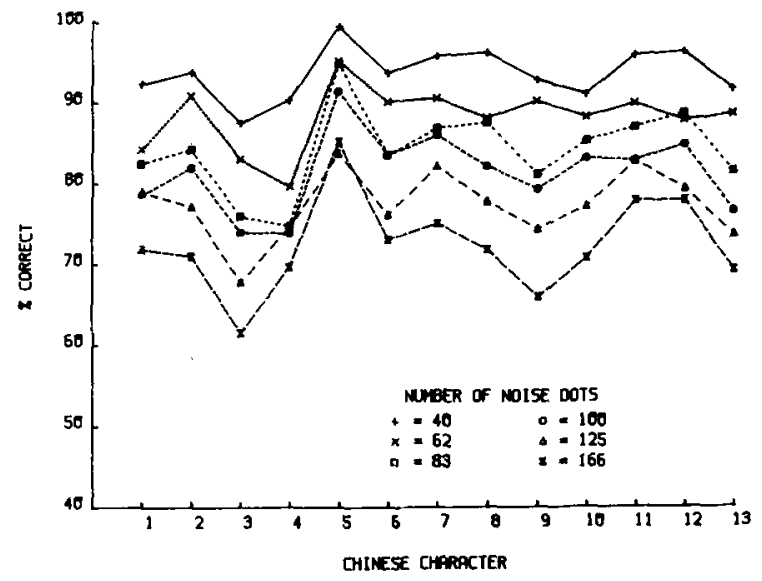

Figure 8. The results of the static-noise experiment plotted as a function of the characters or strokes. The curves are parametric as a function of the density of the noise dots as specified by their number.

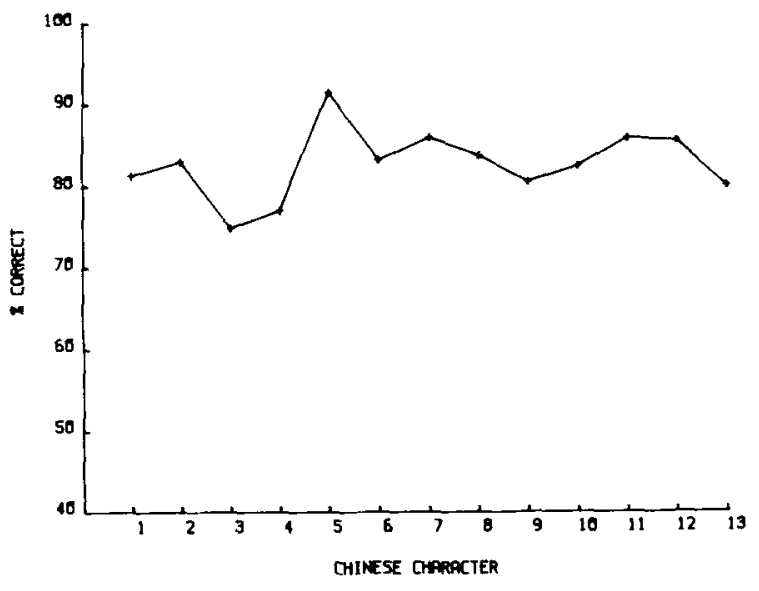

Figure 9. The results of the dynamic-noise experiment pooled for all interdot intervals to display the general trend across the various strokes and characters.

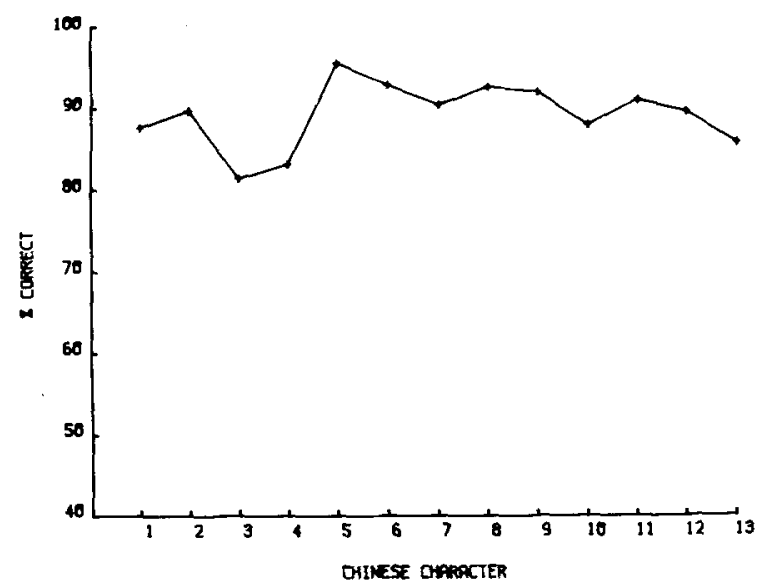

Figure 10. The results of the static-noise experiment pooled for all noise-dot densities to display the general trend across the various strokes and characters.

any of the other stimulus forms. In contrast, and especially remarkable, however, is the fact that the other two single straight-line strokes are only slightly less detectable than the rest of the characters in spite of the fact that there are fewer (6 instead of 14) dots and, for that matter, strokes in these two stimulus forms than in the more complex characters. The only other character that seems to be detected less well relative to the other characters in both Figure 9 and Figure 10 is the one numbered 13. This character is made predominantly from curved strokes and contains no long straight lines. It should be noted that the stimuli numbered $1-4$ are among the most frequently used strokes (Tseng, Chang, \& Chen, 1965) and those numbered 5-13 are among the most frequently used characters in the Chinese written language.

In the following discussion we shall consider the implications of these results to the three questions posed in the introduction. 


\section{DISCUSSION}

The three questions asked in the introduction to this paper can now be answered. The data presented in Figures 4 and 5 speak directly to the possibility that there might have been some cognitive or experiential overtone to the detection task imposed on the subjects in these experiments. This issue is central to our understanding of the level at which these detection processes occur. As noted above, it has generally been assumed that the successful extraction of the stimulus form from the visual noise results from the execution of intermediate-level visual processes that are affected neither by the dynamics of the receptor and the retinal plexus (as long as the individual dots are above absolute threshold) nor by cognitive and experiential factors executed at high nervous levels. The data, in general, support this concept that these relevant processes are intermediate and preattentive-neither very peripheral nor very central. Figure 4 shows that the two groups of subjects performed identically in the dynamicnoise situation. Thus, the familiarity of the Chinesereading subjects was of no advantage. Figure 5 , however, does show a difference between the curves produced by the two groups of subjects. But it is the Chinese-reading subjects who are less proficient for all except the lowest number of dots in the static viewing situation. It may be that this difference is due to the fact that the older Chinesereading subjects are more sensitive to the masking effects of the static dotted noise than are the younger, Chinesepictograph illiterate subjects.

The important fact is that there is no advantage accrued in either experiment to the Chinese-reading subjects as a result of their extensive experience with this type of stimulus material. Of course, even the Chinese-reading subjects were not experienced with dotted pictograms, but some transfer from their experience with solid pictograms would have been expected. Such an assumption in retrospect would obviously have been incorrect. Rather, it seems more likely that it is the actual physical geometry of the dotted stimulus forms that determines their detectability.

This, then, brings us to the next major question. To what degree does the autocorrelation theory (Uttal, 1975), which has been so successful in modeling the detectability of other two-dimensional stimuli, predict the performance of the subjects in this task? To review briefly, the model is based upon the autocorrelational integral:

$$
A c=\iint f(x, y) \cdot f(x+\Delta x, y+\Delta y) d y d x,
$$

where $f(x, y)$ is the stimulus and $f(x+\Delta x, y+\Delta y)$ is a shifted replica of it. Close approximations to the stimuli shown in Figure 1 were autocorrelated with this expression over a $15 \times 15$ space to produce a three-dimensional surface of autocorrelation values for all possible shifted positions $(\Delta x, \Delta y)$. This produces a set of values, or peaks, in the $\Delta x, \Delta y$ space that can be assigned a figure of merit by executing the following expression:

$$
F=\frac{\sum_{n=i}^{N} \sum_{i=j}^{N}\left(A_{i} \times A_{j}\right) / D_{i j}}{N}, \quad i \neq j
$$

where $\mathrm{N}$ is the number of peaks, $\mathrm{D}_{\mathrm{ij}}$ is the pythagorean distance between any two peaks $\mathrm{i}$ and $\mathrm{j}$, and $\mathrm{A}$ is the amplitude of any peak $i$ or $j$. (The complete details of this procedure can be found in Uttal, 1975.)

Application of this autocorrelation transform and the figure-of-merit expressions produces Table 1 -the figures of merit for the 13 stimuli shown in Figure 1. These figures of merit can now be compared with the performance scores on the two experiments when they are analyzed in terms of the 13 characters in the pooled version shown in Figures 9 and 10 for the dynamic and static conditions, respectively.

Before the comparison is made, however, it should be reiterated that the stimuli numbered 1-4 are made up of a single stroke whereas those numbered 5-13 are multiplestroke characters. The fact that stimuli 1 and 2 are detected at approximately the same performance level as are the more densely dotted stimuli numbered 6 through 13 suggests that it is the most visible stroke in each character that is most effective in specifying the level of detectability of these forms. In particular, these two straight lines are detected very well either in isolation or when they are a part of more complex characters. This prepotency of straight lines has been referred to as "the rule of linear periodicity" earlier (Uttal, 1983).

As noted previously, the two most weakly detected stimuli are the ones numbered 3 and 4 in both experiments. They are also the two stimuli with the lowest figure of merit in the model's prediction table. (The great discrepancy between the figures of merit for stimuli numbered $1-4$ and the other stimuli is mainly due to the number of dots-6 vs. 14.)

Proceeding, the one stimulus that is detected to a substantially greater degree than any other (number 5 ) is the only one with a very large figure of merit, as shown in Table 1 . The only other discrepant stimulus form, detected consistently less well than any other in both Figure 9 and

Table 1

The Computed Figures of Merit for 13 Stimuli Used in Both Parts of this Experiment

\begin{tabular}{cc}
\hline Stimulus Number & Figure of Merit \\
\hline 1 & 1513 \\
2 & 1513 \\
3 & 861 \\
4 & 861 \\
5 & 11169 \\
6 & 5873 \\
7 & 5414 \\
8 & 6566 \\
9 & 5458 \\
10 & 5069 \\
11 & 4780 \\
12 & 5081 \\
13 & 4479 \\
\hline
\end{tabular}


Figure 10, is stimulus 13 and, correspondingly, its figure of merit is the lowest of any of the multiple-stroke characters. In general, therefore, the autocorrelation model does a good job of rank ordering the results obtained in this study, even though absolute value comparisons, as usual, are not good predictors.

Finally, we can turn to the third question: Is there any detectability difference attributed to the method-dynamic or static-that was used in the two experiments? The general trends shown in Figures 9 and 10 are similar enough for us to be assured that no distortion vis-à-vis form is introduced by the difference in methods. The effect of form and detectability is well replicated in each of these graphs. On the other hand, the difference between the groups that did obtain, as shown in Figures 4 and 5, may be due to an age-cohort difference between the two groups of subjects and a somewhat greater sensitivity on the part of the older group to the conditions of the static mask condition. Such an age-related decrement in masking performance is well documented elsewhere (e.g., Di Lollo, Arnett, \& Kruk, 1982). These researchers used the interstimulus interval as their dependent variable, but the age function was shown to be steep and nearly linear and could account for this unexpected result in the present experiment. The age effect was not so pronounced in other tasks, however, and this task dependence is also mirrored in the present data.
In summary, however, differences between the two groups of subjects are either small or in the direction opposite to what would have been predicted a priori. This suggests that there is no substantial cognitive or experiential effect on this type of visual process, and dotted form detection is truly an example of an intermediate level of visual processing. Similarly, method does not seem to be important in determining the effect on detectability of the specific stimulus forms used in this experiment. However, there is a substantial effect of stimulus form that is predicted by the autocorrelation model-a model that is passive, automatic, and certainly preattentive.

\section{REFERENCES}

Dilollo, V., Arnett, J. L., \& KRuk, R. V. (1982). Age-related changes in rate of visual information processing. Journal of Experimental Psychology: Human Perception \& Performance, 8, 225-237.

Tseng Hsing-chu, Chang Lu-Hsing, \& Chen Chao-kuan (1965). The relative frequencies of the various stroke-types of the Chinese ideograms. Acta Psychologica Sinica, 3, 2-4.

UTTAL, W. R. (1975). An autocorrelation theory of form detection. Hillsdale, NJ: Erlbaum.

UTTAL, W. R. (1983). Visual form detection in 3-dimensional space. Hillsdale, NJ: Erlbaum.

UTTAL, W. R. (1984). The detection of nonplanar surfaces in visual space. Hillsdale, NJ: Erlbaum.

(Manuscript received April 10, 1985; revision accepted for publication July 29, 1985.) 